\title{
Dendrigraft of Poly-L-lysine as a Promising Candidate to Reverse Heparin-based Anticoagulants in Clinical Settings
}

\author{
Benjamin Ourri, ${ }^{a}$ Jean-Patrick Francoia, ${ }^{b}$ Gerald Monard, ${ }^{\star c}$ Jean-Christophe Gris, ${ }^{\text {*d,e }}$ \\ Julien Leclaire ${ }^{a}$ and Laurent Via|*a;b
}

\begin{abstract}
a Univ. Lyon, Université Claude Bernard Lyon 1, ICBMS UMR CNRS 5246, 43 Boulevard du 11 Novembre 1918, 69622 Villeurbanne Cedex, France. E-mail: laurent.vial@univ-lyon1.fr

${ }^{b}$ Institut des Biomolécules Max Mousseron, UMR 5247 CNRS - Université de Montpellier - ENSCM, Place Eugène Bataillon, 34296 Montpellier Cedex 5, France

' Univ. Lorraine, LPCT UMR 7019 CNRS, Boulevard des Aiguillettes B.P. 70239, 54506 Vandoeuvre-les-Nancy, France, France. E-mail: gerald.monard@univ-lorraine.fr

d Department of Hematology, Nîmes University Hospital, University of Montpellier, 30029 Nîmes Cedex 9, France. E-mail: jean.christophe.gris@chu-nimes.fr

${ }^{\mathrm{d}}$ The First I.M. Sechenov Moscow State Medical University, Russian Federation

${ }^{*}$ Corresponding authors
\end{abstract}

\section{General}

All commercial chemicals were used as received without further purification. DGLs were provided by the COLCOM company. UV / absorbance measurements were performed on a VICTOR Nivo Multimode Microplate Reader at $25^{\circ} \mathrm{C}$. Anti-Xa assays were performed on a STA ${ }^{\circledR}-R$ Max automated analyzer from Stago. GPU resources were allocated by the mésocentre EXPLOR of the Université de Lorraine.

\section{Indicator-displacement assays}

For the purpose of calculations, the molecular weight of UFH is assumed as that of the sodiated analogue of the heparin repeat unit (i.e. $665.40 \mathrm{~g} \cdot \mathrm{mol}^{-1}$ ). It should be noted that as supplied, UFH only contains ca. $30-40 \%$ of material with the active sequence of repeat units. However, all of the sample contains anionic saccharide units which can bind, even if they are in the wrong sequence. Hence to best evaluate binding concentrations and charge efficiencies, we report the total concentration of the anionic disaccharide - irrespective of whether it is present in the active form of heparin or not.

In a typical experiment, microplate wells were filled with $81 \mu \mathrm{M}$ (final concentration) of UFH, $67.5 \mu \mathrm{M}$ (final concentration) of Azure $A$ and increasing concentrations of antidote. All solutions were prepared in a Phosphate Buffered Saline (PBS 1X) at pH 7.4. The absorbance was measured $592 \mathrm{~nm}$ at $25^{\circ} \mathrm{C}$, and the reported values were a mean of triplicate experiments.
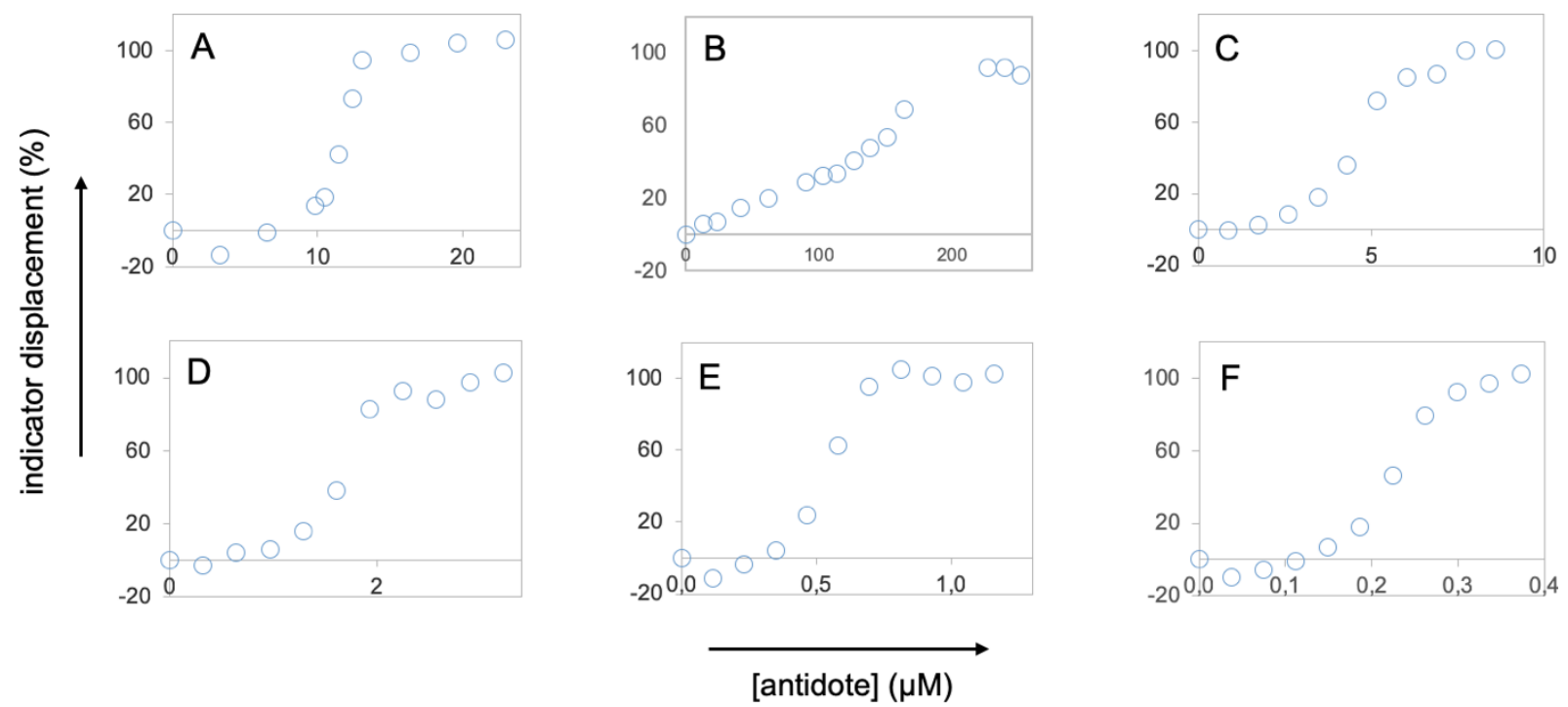

Figure S1 - UFH binding curves in PBS $1 X$ from the displacement of Azure A with (A) protamine; (B) DGL G1; (C) DGL G2; (D) DGL G3; (E) DGL G4; (F) DGL G5. 


\section{Anti-Xa assays}

All blood samples were obtained by clean venipuncture performed between 8:00 and 10:00 a.m. after overnight fasting in clinically normal individuals not taking any drugs, consulting in our Outpatient Department of Haematology, University Hospital, Nîmes, for systematic medical check-up and biological screening including coagulation tests. Samples were collected in 1/10 volume of CTAD anticoagulant-antiplatelet mixture $(0.109 \mathrm{M}$ trisodium citrate, pH 5.4; theophylline; $3.7 \mathrm{mM}$ adenosine; $0.198 \mathrm{mM}$ dipyridamole; Diatube $\mathrm{H}$; Becton Dickinson, Rungis, France) after systematic rejection of the first $2 \mathrm{~mL}$ of blood. Following a double centrifugation at $4000 \times \mathrm{xg}$ for 20 minutes, aliquots of platelet-poor plasma were controlled for normal coagulation screening tests (aPTT ratio $<1.2$, PT ratio $<1.2$, fibrinogen 2 - $3.5 \mathrm{~g} / \mathrm{L}$, no detectable anti-Xa plasma activity) and residual plasma material was immediately stored at $-80^{\circ} \mathrm{C}$. Forty individual samples were thus prepared, sex-ratio $1: 1$, median age 29 years (22-48). Just before use, the frozen plasmas were thawed by quick immersion for 5 minutes in a $37^{\circ} \mathrm{C}$. water-bath, and then systematically homogenized by vortexing. They were finally mixed to produce the final normal pooled plasma NPP. Dilutions of heparin-related, clinically-relevant anticoagulants (unfractionated heparin UFH: Heparine ${ }^{\circledR}$ Roche, low-molecular weight heparin LMWH: enoxaparin, Lovenox ${ }^{\circledR}$, pentasaccharide: fondaparinux, Arixtra ${ }^{\circledR}$ ) were serially performed in $0.9 \% \mathrm{NaCl}$ then subsequently mixed with NPP, at a fixed dilution rate: 1 volume / 9 volumes NPP, in order to produce samples to be tested for the inhibition of the heparin-related anti-Xa activity.

Anti-Xa plasma activities were assayed by a commercially-available chromogenic test (STA ${ }^{\circledR}$-Rotachrom, Diagnostica Stago, Asnières, France), which quantifies the inhibition of an added fixed amount of purified factor Xa enzyme by antithrombinheparin complexes present in the tested plasma samples. Relevant calibration curve plasmas and control plasmas were also from Stago. All tests were driven on the STA ${ }^{\circledR}-\mathrm{R}$ Max automated analyzer from Stago.

\section{Computational Methods}

Molecular modeling and simulations of DGLs, protamines, heparins and their corresponding complexes have been performed using the AMBER biomolecular package. ${ }^{\mathrm{S} 1}$ Eight DGL samples structures were prepared as detailed in Ref. S2. Their primary structures are presented in Table S1. Four protamine samples have been built. Their primary structures are presented in Table S2. All these proteins have been modeled with the AMBER ff14SB protein force field, ${ }^{\text {S3 }}$ whereas the GLYCAM06 carbohydrate force field ${ }^{54}$ was used for UFH, enoxaparin, and fondaparinux sugar molecules. After an initial $10 \mu$ second molecular dynamics in implicit water solvent of each individual component, ${ }^{\mathrm{S} 2} \mathrm{DGL} /$ heparin and protamine/heparin complexes have been built in explicit TIP3P water. ${ }^{5}$ Systems were solvated in truncated octahedrons with a minimum distance between any solute atom and the edge of the periodic box of $20 \AA$, yielding systems ranging from around 62,000 to about 764,000 atoms depending on the size and the initial shape of the complexed moieties. Each system was neutralized by adding the right amount of counter sodium or chloride ions.

First, all molecular systems have been energy minimized for 10,000 steps using consecutively 1,000 steps of steepest descent followed by 9,000 steps of conjugate gradients. Then they have been heated from 10 to $300 \mathrm{~K}$ during $250 \mathrm{ps}$ at constant pressure ( $1 \mathrm{bar}$ ) with a time step of $1 \mathrm{fs}$. A $20 \mathrm{kcal} / \mathrm{mol} / \AA^{2}$ restrained harmonic potential was applied on all non-hydrogen atoms during heating to prevent the loss of the initial three-dimension complexed shape. Productions runs were performed for 2 microseconds, except for the protamine/UFH complexes whose sizes are comprised between 380,000 and 764,000 atoms and for which production molecular dynamics runs were limited to 1 microsecond.

All molecular dynamics simulations were carried out using the GPU implementation of the pmemd program in AMBER18. ${ }^{56}$ For all equilibration and production runs, the Particle Mesh Ewald summation technique was used with the default $8 \AA$ cutoff. Bonds involving hydrogen atoms were constrained by the SHAKE algorithm. ${ }^{57}$ Temperature was controlled with a Langevin thermostat at $300 \mathrm{~K}$ with a collision frequency $Y=10.0 \mathrm{ps}^{-1}$. Constant pressure dynamics at 1 bar was applied with isotropic position scaling and controlled by a Monte Carlo barostat with volume change attempts every 100 steps. For production runs, a time step of $4 \mathrm{fs}$ was used with hydrogen mass repartitioning. ${ }^{8}$

All trajectory analyses were carried out using the cpptraj module of AMBER. ${ }^{59}$

The free energies of binding between any DGL or protamine on one side and any heparin on the other side were computed using the equation:

$\Delta G_{\text {binding }}=\langle G P H\rangle-\langle G P\rangle-\langle G H\rangle$

where $\mathrm{PH}, \mathrm{P}$ and $\mathrm{H}$ stand for protein-heparin complex, protein (i.e. either DGL or protamine), and heparin (i.e. UFH, enoxaparin, or fondaparinux), respectively. The average free energy of each system is estimated as a sum of three terms:

$G=E_{M M}+G_{S O l v}-T S_{M M}$ 
where $E_{M M}$ is the molecular mechanics energy of each system, including internal, non-bonded electrostatics, and van der Waals energies. $G_{s o l v}$ is the solvation energy which consists of a polar and a non-polar part. The polar solvation free energy is calculated by a Generalized Born (GB) approach. The nonpolar solvation free energy is computed by a relation to the solvent-accessible surface area (SASA). The last term TSMM is the product of absolute temperature and the entropy.

In this study, the first two terms were calculated using the MMPBSA.py module of AMBER18 with all water and counter-ions stripped off. ${ }^{510}$ To evaluate the polar solvation free energy, the GBobc solvation model was used. ${ }^{511}$ The hydrophobic contribution has been approximated by the Linear Combinations of Pairwise Overlaps (LCPO) method. ${ }^{\text {S12 }}$ The entropy term was not included in our calculations. Preliminary studies on a few protein/heparin complexes (data not shown) using normalmode analysis showed us that the entropy contribution was small as compared to the other components of the free energy of binding whereas the computer times were increased by a large factor. ${ }^{\mathrm{S13}}$

Table S1: DGL G2 primary sequences (see ref. S2 for nomenclature details)

G2-1 : ACDDDDDDCCCCh 8CCCCCCCCh 7CCCCCCCCh 6CCCh 5CCh 4CCCCCCh 3CCh

G2-2 : ADDDDCDDCCCCCCh 8CCCCh 7CCCCCCh 5CcCcccCh 4CCCCh 3CcCCh 2CCh

G2-3: BCDCDDDDCCh 8CCCCCh 7CCCCCCCCCh 6CCCCCh 5CCCCh 3CCCCh 1CCCCh

G2-4 : BDCDCDDDCCh 8CCCCCCh 7CCCCCCCh 6CCCCCCh 4CCCCh 2CCCh 1CCCCCh

G2-5: BDCDDDDCCCCh 7CCCCCCCCh 6CCCCh 5CCCCCh 4CCCCh 2CCCCCCh 1CCCh

G2-6 :: BDDCDDDCCCCh 7CCCCCh 6CCCCCCCh 5CCh 3CCCCCCCCCh 2CCCCCh 1CCh

G2-7 : BDDDCCDDCCCCCCh 8CCCCh 7CCCCCCCh 4CCCh 3CCCCCCCh 2CCCh 1CCCh

G2-8: BDDDCDDCCCCCCCCh 7CCCh 6CCh 4CCh 3CCCCCCCCh 2CCCCCCh 1CcccCh

Table S2 : Protamine primary sequences

P1 : PRRRRRSSSR PIRRRRRPRA SRRRRRGGRR RR

P2 : PRRRRSSRRP VRRRRRPRVS RRRRRRGGRR RR

P3 : PRRRRSSSRP VRRRRRPRVS RRRRRRGGRR RR

P4 : PRRRRASRRI RRRRRPRVSR RRRRGGRRRR 


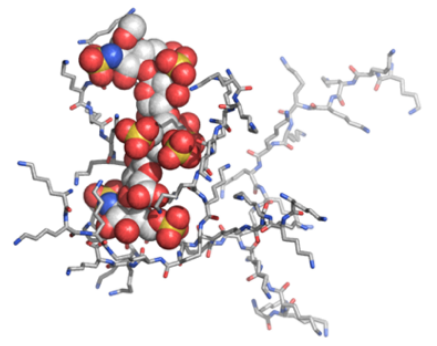

DGL G2-1:fondaparinux

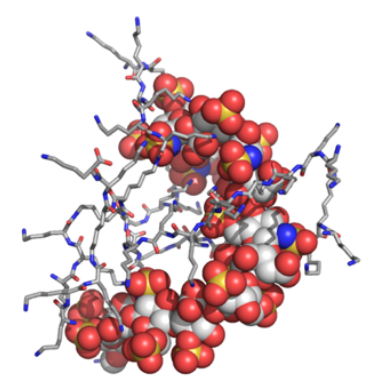

DGL G2-1:enoxaparin

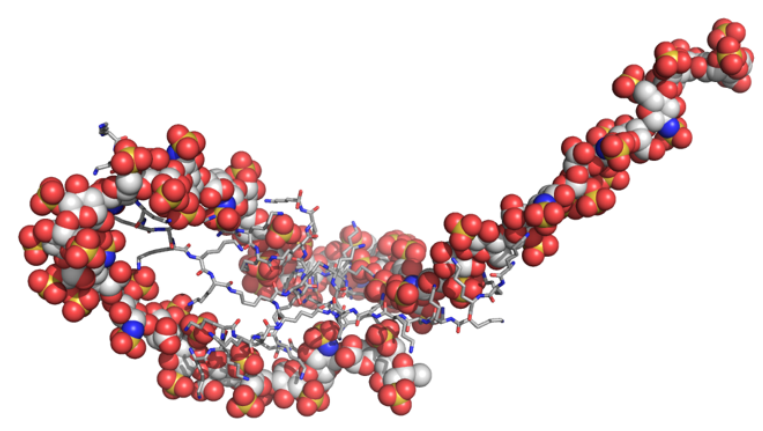

DGL G2-1:UFH

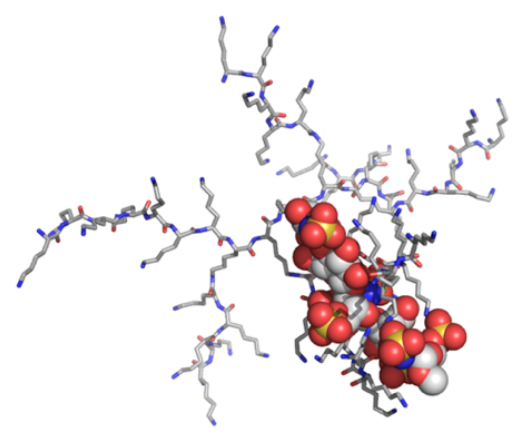

DGL G2-2:fondaparinux

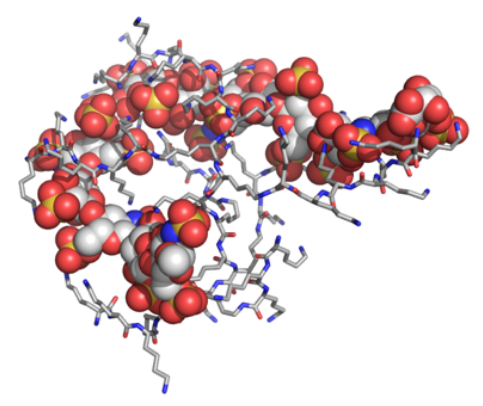

DGL G2-2:enoxaparin

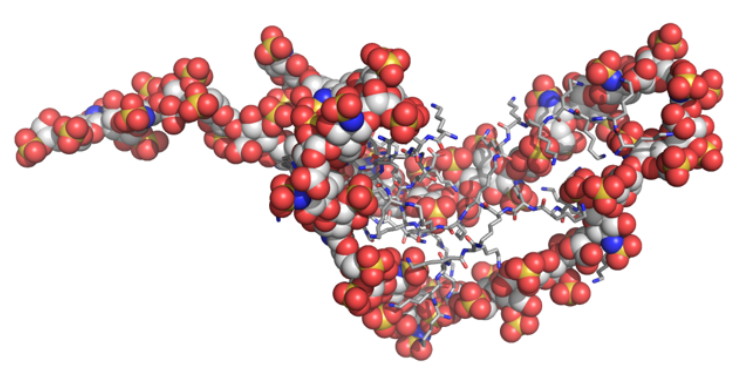

DGL G2-2:UFH

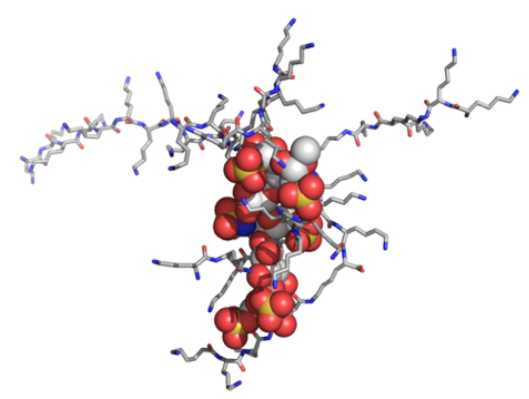

DGL G2-3:fondaparinux

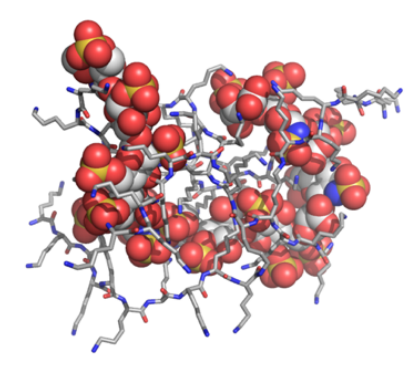

DGL G2-3:enoxaparin 


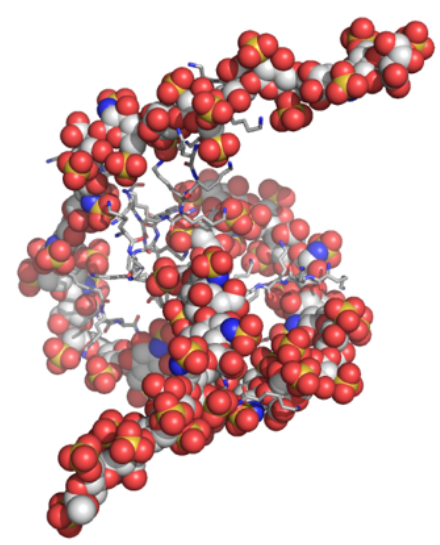

DGL G2-3:UFH

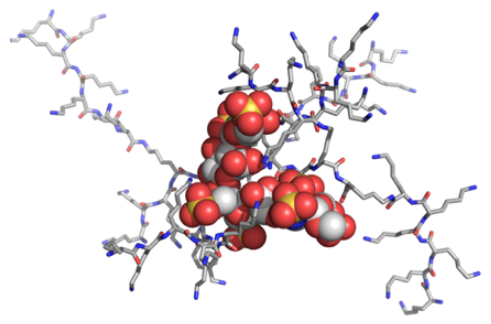

DGL G2-4:fondaparinux

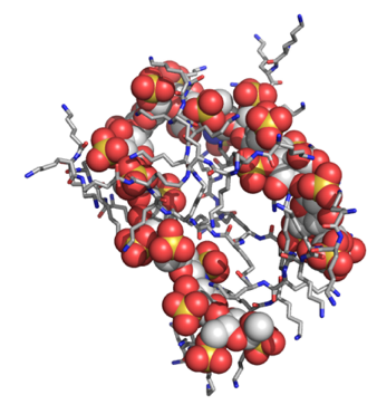

DGL G2-4:enoxaparin

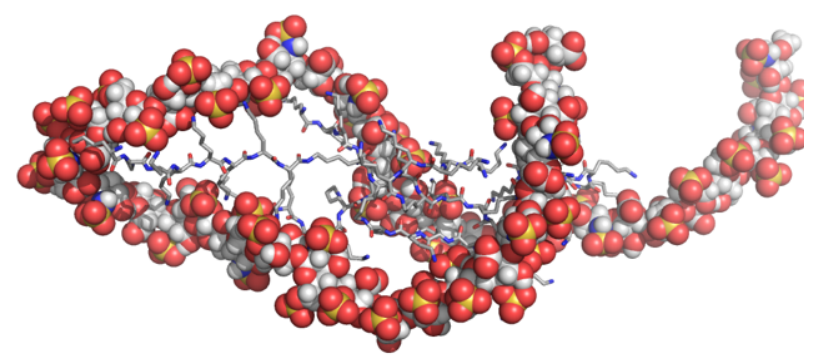

DGL G2-4:UFH

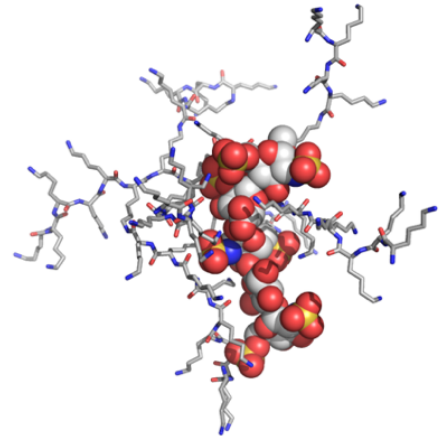

DGL G2-5:fondaparinux

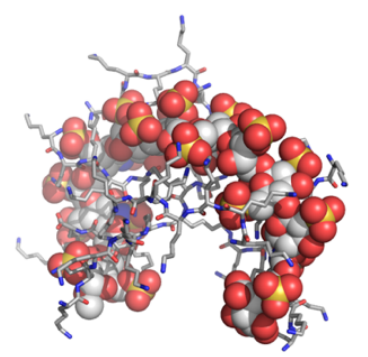

DGL G2-5:enoxaparin

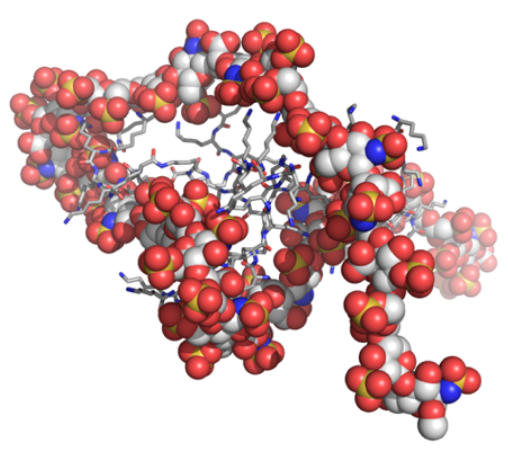

DGL G2-5:UFH

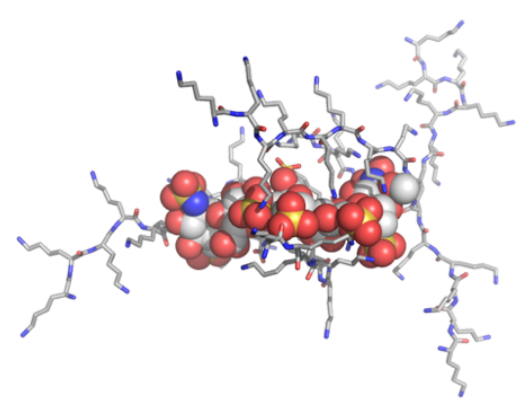

DGL G2-6:fondaparinux 


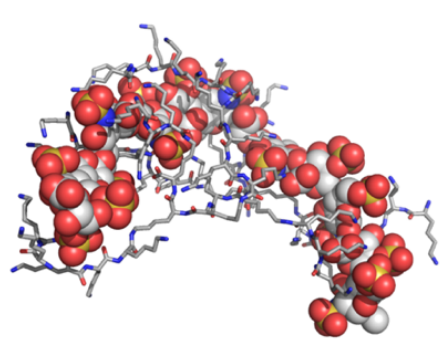

DGL G2-6:enoxaparin

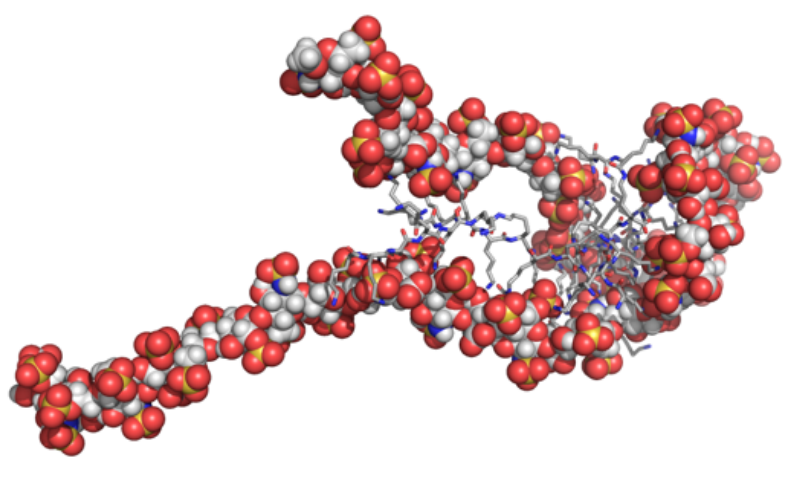

DGL G2-6:UFH

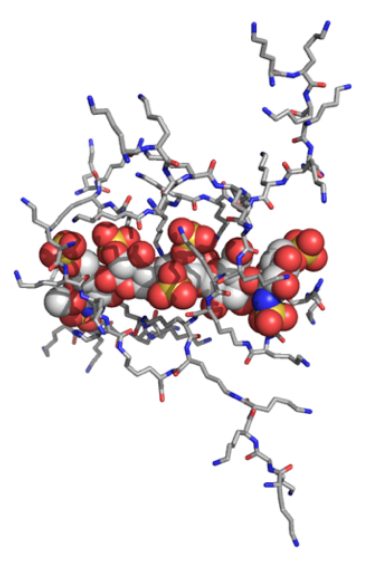

DGL G2-7:fondaparinux

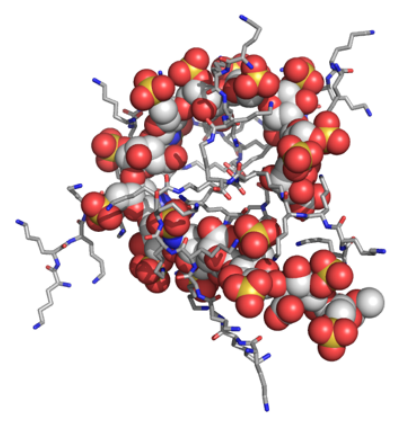

DGL G2-7:enoxaparin

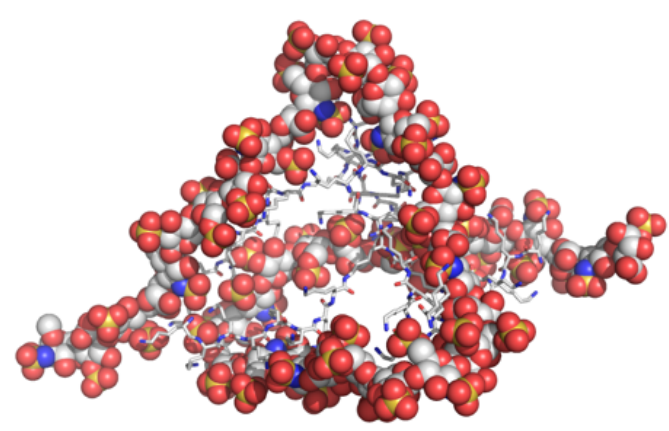

DGL G2-7:UFH

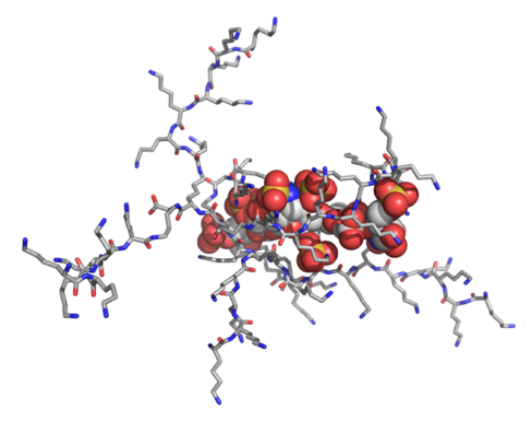

DGL G2-8:fondaparinux

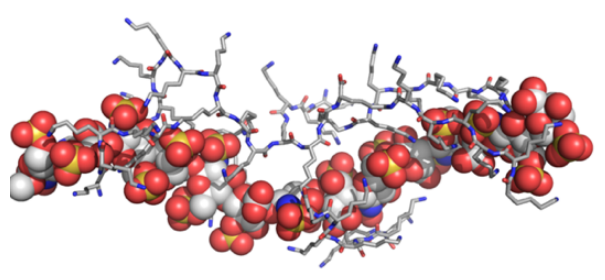

DGL G2-8:enoxaparin

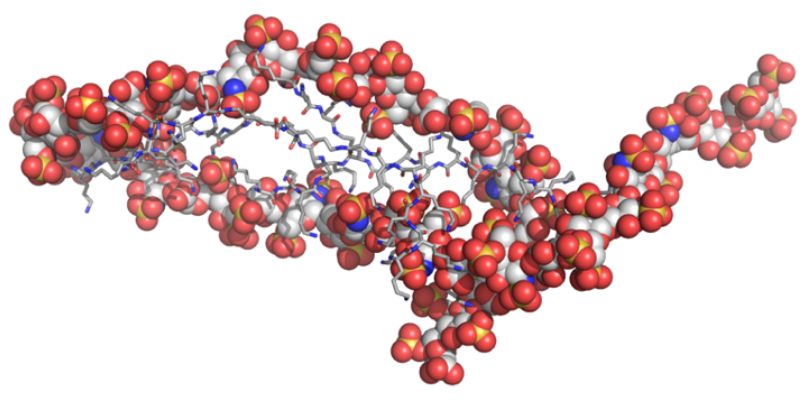

DGL G2-8:UFH 


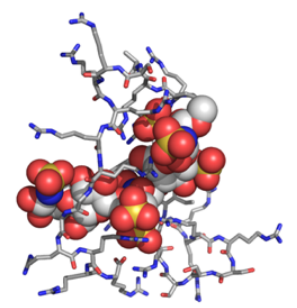

protamine $\mathbf{P 1}$ :fondaparinux

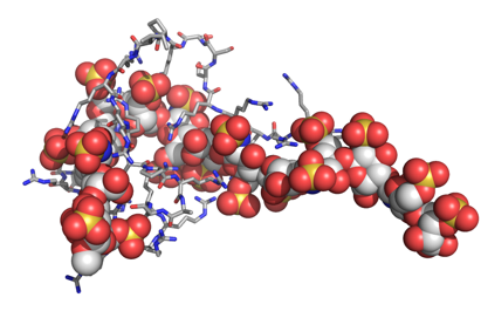

protamine $\mathbf{P 1}$ : enoxaparin
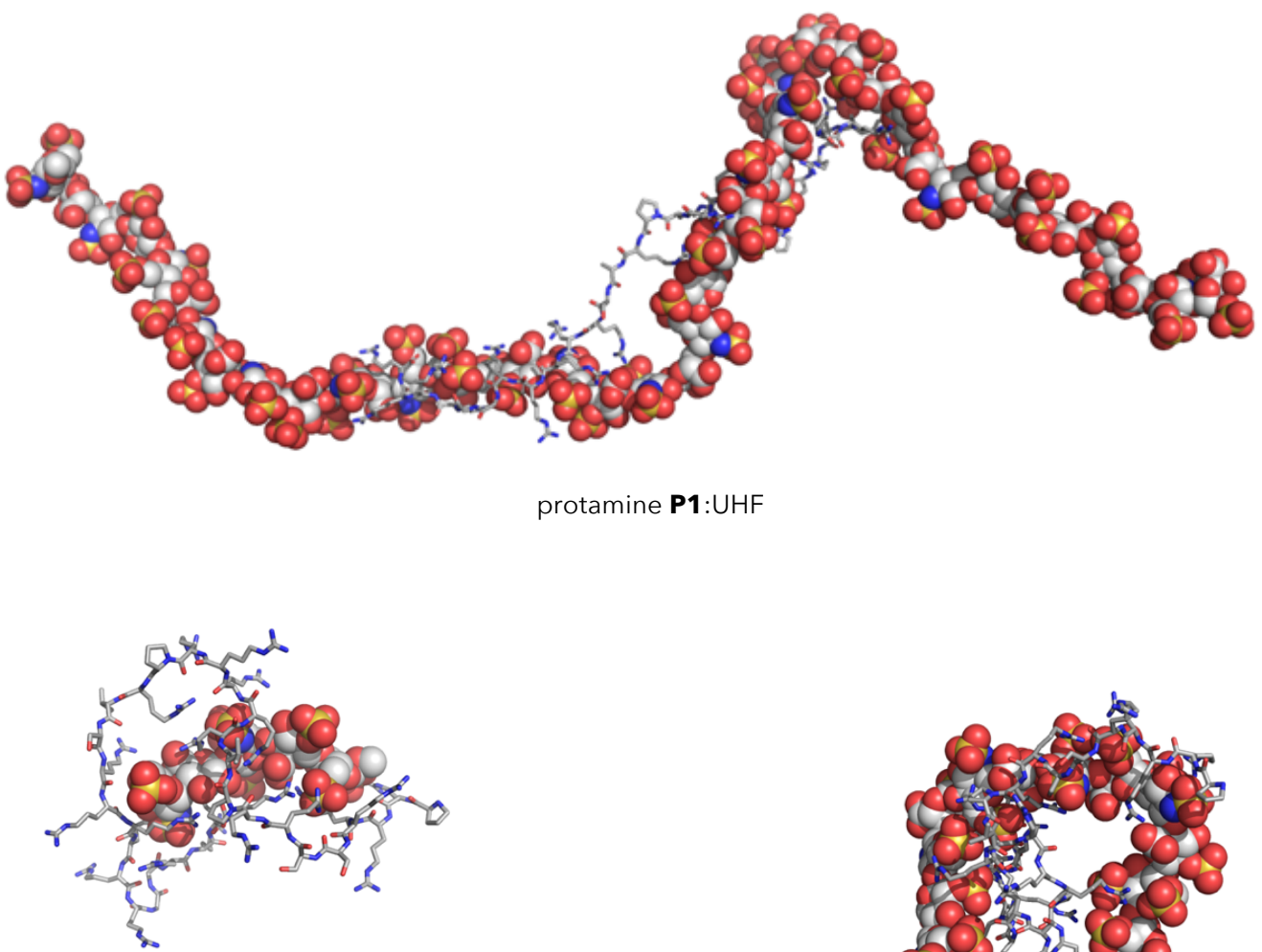

protamine P2:fondaparinux

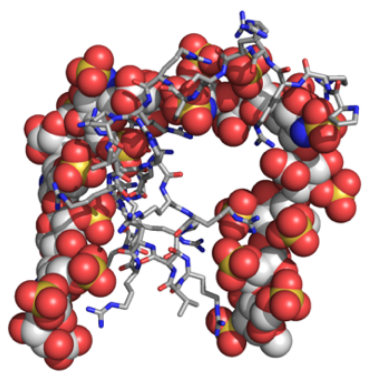

protamine P2: enoxaparin

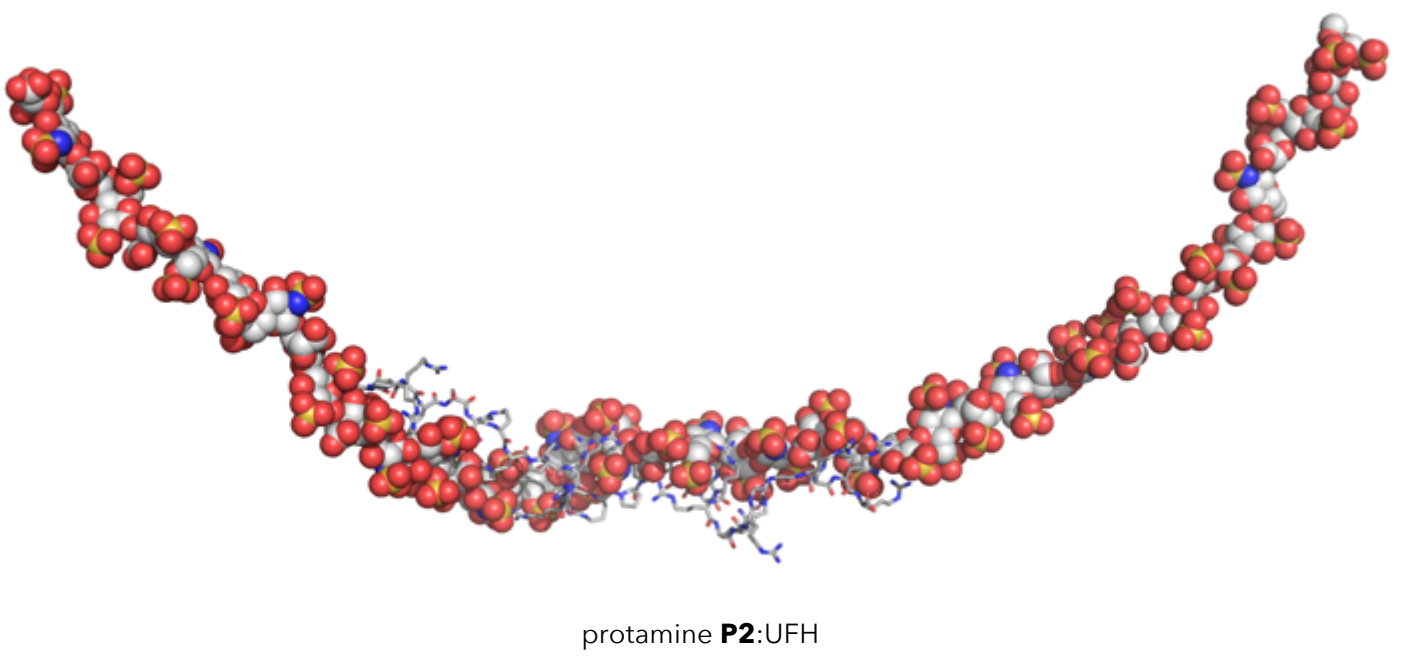




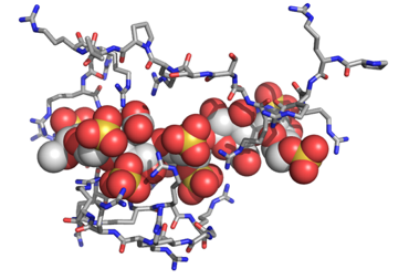

protamine $\mathbf{P 3}$ :fondaparinux

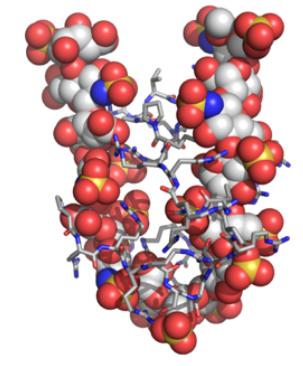

protamine $\mathbf{P 3}$ :enoxaparin

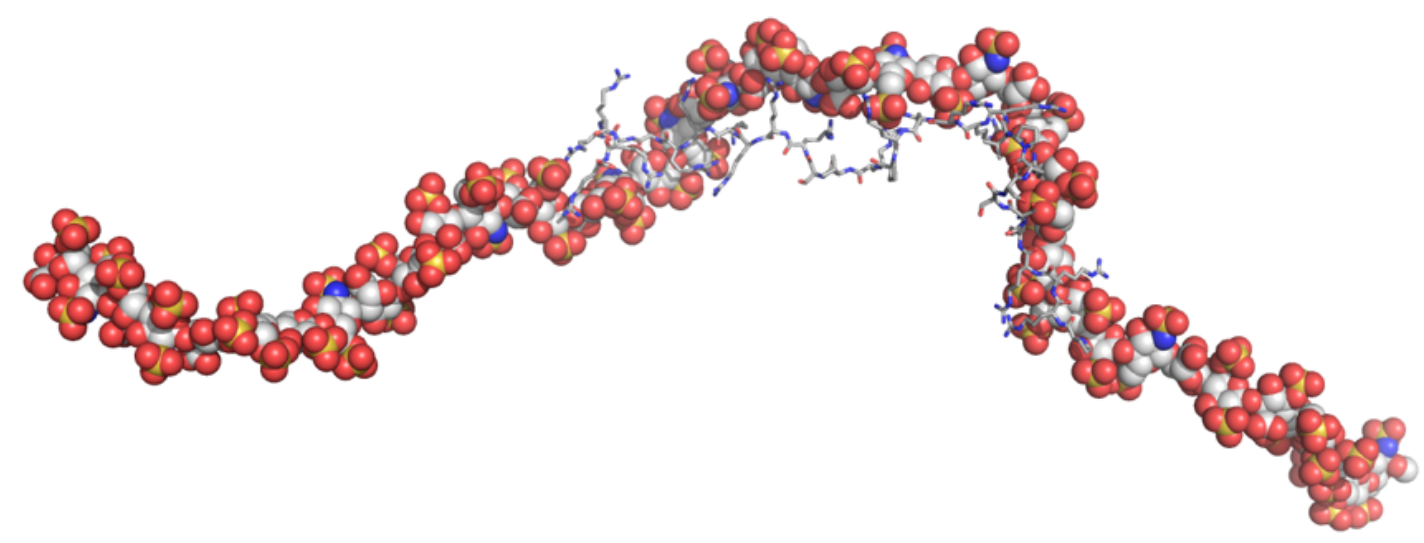

protamine $\mathbf{P 3}$ :UFH

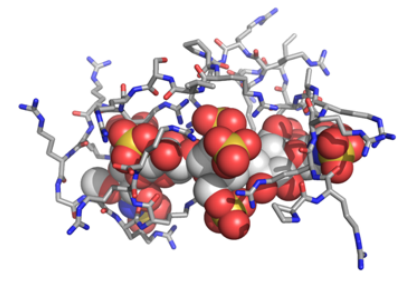

protamine P4:fondaparinux

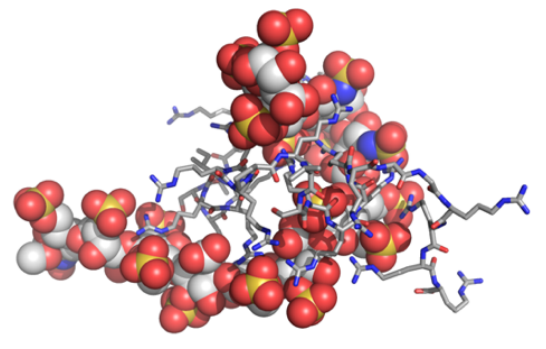

protamine P4:enoxaparin

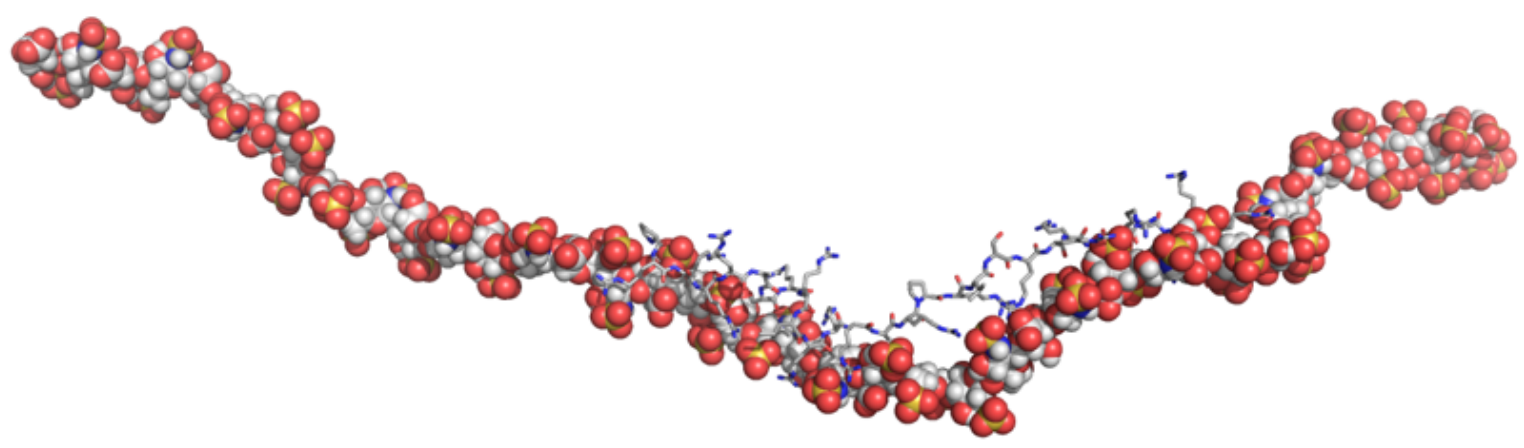

protamine P4:UFH 
Figure S2 - Snapshots taken at the end of the MD trajectories for the stoichiometric complexes DGL G2:fondaparinux, DGL G2:enoxaparin, DGL G2:UFH, protamine:fondaparinux, protamine:enoxaparin, and protamine:UFH. Heparins are represented as balls, and antidotes as sticks.

\section{References}

${ }^{51}$ Case, D. A.; Brozell, S. R.; Cerutti, D. S.; T. E. Cheatham, I.; Cruzeiro, V. W. D.; Darden, T. A.; Duke, R. E.; Ghoreishi, D.; Gohlke, H.; Goetz, A. W.; Greene, D.; Harris, R.; Homeyer, N.; Izadi, S.; Kovalenko, A.; Lee, T. S.; LeGrand, S.; Li, P.; Lin, C.; Liu, J.; Luchko, T.; Luo, R.; Mermelstein, D. J.; Merz, K. M.; Miao, Y.; Monard, G.; Nguyen, H.; Omelyan, I.; Onufriev, A.; Pan, F.; Qi, R.; Roe, D. R.; Roitberg, A.; Sagui, C.; Schott-Verdugo, S.; Shen, J.; Simmerling, C. L.; Smith, J.; Swails, J.; Walker, R. C.; Wang, J.; Wei, H.; Wolf, R. M.; Wu, X.; Xiao, L.; York, D. M.; Kollman, P. A. AMBER 2018. University of California: San Francisco, 2018.

S2 Francoia, J.-P.; Rossi, J.-C.; Monard, G.; Vial, L. Digitizing Poly-L-Lysine Dendri- grafts: From Experimental Data to Molecular Dynamics Simulations. Journal of Chemical Information and Modeling 2017, 57, 2173-2180.

s3 Maier, J. A.; Martinez, C.; Kasavajhala, K.; Wickstrom, L.; Hauser, K. E.; Simmer- ling, C. ff14SB: Improving The Accuracy of Protein Side Chain and Backbone Pa- rameters from ff99SB. J. Chem. Theory Comput. 2015, 11, 3696-3713.

${ }^{54}$ Kirschner, K.; Yongye, A.; Tschampel, S.; Gonza'lez-Outeiriñ, J.; Daniels, C.; Fo- ley, B.; Woods, R. GLYCAM06: A generalizable biomolecular force field. Carbohy- drates. J. Comput. Chem. 2008, 29, 622-655.

s5 Jorgensen, W. L.; Chandrasekhar, J.; Madura, J. D.; Impey, R. W.; Klein, M. L. Com- parison Of Simple Potential Functions For Simulating Liquid Water. J. Chem. Phys. 1983, 79, 926-935.

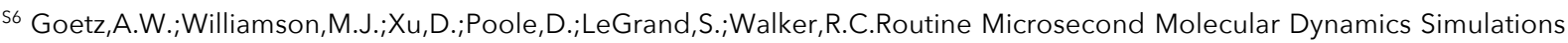
with AMBER on GPUs. 1. General- ized Born. J. Chem. Theory Comput. 2012, 8, 1542-1555.

${ }^{57}$ Ryckaert, J.-P.; Ciccotti, G.; Berendsen, H. J. Numerical Integration of the Carte- sian Equations of Motion of a System with Constraints: Molecular Dynamics of n-alkanes. J. Comput. Phys. 1977, 23, 327-341.

${ }^{58}$ Hopkins,C.W.;LeGrand,S.;Walker,R.C.;Roitberg,A.E.Long-Time-StepMolecu- lar Dynamics through Hydrogen Mass Repartitioning. J. Chem. Theory Comput. 2015, 11, 1864-1874.

s9 Roe, D. R.; Cheatham III, T. E. PTRAJ and CPPTRAJ: Software for Processing and Analysis of Molecular Dynamics Trajectory Data. J. Chem. Theory Comput. 2013, 9, 3084-3095.

${ }^{510}$ Miller III, B. R.; McGee Jr., T. D.; Swails, J. M.; Homeyer, N.; Gohlke, H.; Roit- berg, A. E. MMPBSA.py: An Efficient Program for End-State Free Energy Calcula- tions. J. Chem. Theory Comput. 2012, 8, 3314-3321.

S11 Onufriev, A.; Bashford, D.; Case, D. Exploring protein native states and large-scale conformational changes with a modified generalized Born model. Proteins 2004, 55, 383-394.

\$12 Weiser, J.; Shenkin, P.; Still, W. Approximate Atomic Surfaces from Linear Combi- nations of Pairwise Overlaps (LCPO). J. Computat. Chem. 1999, 20, 217-230.

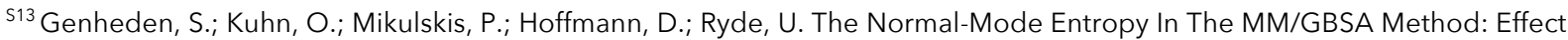
Of System Truncation, Buffer Region, And Dielectric Constant. J. Chem. Inf. Model. 2012, 52, 2079-2088. 